\title{
INTEGRAÇÃO ENTRE EPIDEMIOLOGIA E INTERNET
}

\author{
INTEGRATION BETWEEN EPIDEMIOLOGY AND INTERNET \\ INTEGRACIÓN ENTRE EPIDEMIOLOGÍA E INTERNET
}

\author{
Sérgio Ribeiro dos Santos ${ }^{1}$ \\ Lenilde Duarte de Sá ${ }^{2}$
}

\begin{abstract}
RESUMO: O presente estudo tem como objetivo apresentar algumas reflexões teóricas sobre a Internet como uma ferramenta de integração no serviço de epidemiologia. Nessa perspectiva, focaliza a epidemiologia e a Internet, a Internet e a pesquisa cooperativa, e, por fim, a importância da Internet no processo de comunicação epidemiológica. A integraçāo entre o saber epidemiológico e o uso da Internet para divulgaçāo do conhecimento é enfatizada como um dos maiores beneficios dessa tecnologia que globaliza a informação e transcende as formas tradicionais de comunicação. Todavia, ressalta-se que essa tecnologia, por si só, näo é a solução para resolver os problemas funcionais dos sistemas de informação em saúde.
\end{abstract}

PALAVRAS-CHAVE: internet, epidemiologia, informaçăo em saúde

\section{INTRODUÇÃO}

A Internet se constitui atualmente em um novo veículo de comunicação que interliga o mundo e globaliza o acesso à informação rápida e precisa. A humanidade está mergulhada numa fantástica forma de comunicação que poderá mudar radicalmente estilos de vida, processos de trabalho e até mesmo o relacionamento entre as pessoas. Apesar do seu sucesso, a Internet não é novidade. Surgiu na década de 60 , criada pelos norte-americanos para servir como guia de comunicação durante a guerra fria entre os Estados Unidos e a extinta União Soviética. Com o término desse periodo obscuro da história, a rede perdeu sua utilidade original e passou a ser instrumento de pesquisa entre universidades.

A partir dos anos de 1990, a Internet começou a adquirir as caracteristicas e as dimensões que a estão transformando na maior rede de comunicação do mundo. O fenômeno Internet está revolucionando a estrutura organizacional e o ambiente domiciliar, otimizando processos, agregando valores, reduzindo erros, proporcionando segurança e precisão, bem como garantindo a democratizaçăo cada vez maior da informaçăo. Além disso, através de seu uso, não é necessário sair de casa para conhecer e descobrir o que o mundo tem a oferecer, em termos de informação e entretenimento.

A Internet possibilita que as pessoas tenham contatos interativos, façam novas amizades, conheçam cidades, culturas e troquem idéias e experiências. A expectativa para o novo milênio que se inicia é a construção de esquemas avançados de mecanismos tecnológicos, capazes de fazer as pessoas se comunicarem simultaneamente em tempo real e no seu idioma nativo. Sua aplicabilidade também pode permitir o acesso à base de dados, em treinamentos ou ensino a distância on line, no envio e recebimento de documentos, workgroups e virtual workgroup, incluindo som e animação, o correio eletrônico, a transferência de imagens, textos e programas

\footnotetext{
' Mestre em Enfermagem. Professor do Departamento de Enfermagem-DEMCA. Doutorando em Ciências da Saúde da UFPB.

${ }^{2}$ Doutora em Enfermagem. Professora do Departamento de Enfermagem-DESPP/UFPB.
} 
entre quaisquer computadores lígados à rede, revolucionando, assim, a ciência em geral. $\mathrm{Na}$ área de saúde o emprego da Internet também tem se mostrado promissor, com o surgimento de sites que prestam serviços, informações, marcam e até realizam consultas on line. Mas, é na epidemiologia que a Internet pode tornar-se uma ferramenta extremamente necessária nas instituiçőes públicas e privadas de saúde, não só pelos beneficios que apresenta, mas também devido ao crescimento progressivo de informaçōes importantes e até indispensáveis aos profissionais que lidam com a epidemiologia.

De acordo com Sabbatini (1993), se não mudarmos radicalmente a forma de acesso à informação, como a geramos, como a distribuimos e como a utilizamos, vamos fatalmente nos afogar nesse maremoto irresistivel de informação. O progresso tecnológico na área de informática é rapidissimo e os epidemiologistas sabem que, sem esse conhecimento, năo será possivel continuar avançando na busca da ciência.

O impacto profundo da Internet sobre a epidemiologia se revela no campo da pesquisa, por ser uma fonte na busca de dados para análise e desenvolvimento de estudos que venham contribuir para o conhecimento mais apurado dos fenômenos epidemiológicos que podem estar disponiveis na rede de comunicação. Essa forma de investigação epidemiológica, via Internet, faz surgir o que denominamos de cultura digital em saúde, tendo em vista a sua progressiva incorporação nos processos técnicos e aplicações epidemiológicas, pela grande quantidade de publicaçōes técnicas e matérias disponiveis na web sites, assim como pelo aumento expressivo de pesquisas nos últimos anos. Todo este potencial deve-se às caracteristicas técnicas da Internet que torna mais fácil o intercâmbio de informações entre a ciência (representada pelos pesquisadores) e o público ou usuário (representado pelo senso comum).

Graças à capacidade da Internet em disponibilizar serviços como o e-mail e o WWW (World Wide Web) ou o FTP (File Transfer Protocol) - Protocolo para Transferência de Arquivos - os epidemiologistas podem manter contato mais fácil e rápido com as unidades sanitárias, enviando e recebendo mensagens através de e-mail ou correio eletrônico. Além disso, o WWW permite a transmissão de arquivos denominados de hipertexto que se utiliza do protocolo HTTP (Hyper Text Transfer Protocol).

Portanto, a questão principal analisada neste estudo envolve uma reflexão sobre a importância da Internet na epidemiologia. Assim, o nosso objetivo é mostrar que a Internet é uma importante ferramenta de integração no serviço da epidemiologia.

\section{EPIDEMIOLOGIA E INTERNET}

A palavra epidemiologia deriva de epidemia, que, na tradução literal do grego, significa sobre a população. Epidemiologia é o estudo da distribuição e da dinâmica das doenças nas populaçōes humanas (Araújo, 1983). Para Breilh e Granda (1989), o método epidemiológico estuda o processo saúde-doença em sua dimensão social.

O conceito de epidemiologia sofreu alteraçōes ao longo dos anos. De acordo com os novos conhecimentos cientificos e transformações sociais e ambientais, a epidemiologia foi se adaptando e incorporando novos conceitos. Para Susser e Susser (1998), a tecnologia pode conduzir a epidemiologia a um novo paradigma que reside, por um lado, na biologia e nas técnicas biomédicas e, por outro, nos sistemas de informação. Essas mudanças têm produzido enorme impacto nas situaçōes de perfis epidemiológicos, em virtude do desenvolvimento sócioeconômico e tecnológico, bem como das reformulaçōes impostas pelas disciplinas da área de saúde em seus diversos campos de atuação que demonstram uma relevante participação da epidemiologia nos processos de intervenção social.

Nesse contexto, a Internet aos poucos vem se incorporando aos sistemas de informação epidemiológica causando um expressivo impacto no dia-a-dia dos pesquisadores e das pessoas, pela facilidade de repassar a informação em tempo real. Assim, estando os processos de 
decisão melhor informados, propiciam maior potencialidade de serem efetivamente concretizados pelos epidemiologistas.

Nesse sentido, reconhece-se a necessidade de que o desenvolvimento da tecnologia da Internet e a epidemiologia estejam integrados num plano maior de comunicação global para democratizar a informação em saúde. Para tanto, faz-se necessário que os políticos e os gestores dos órgãos governamentais, responsáveis pela saúde pública, sejam convencidos das vantagens de conectar toda a rede pública de saúde à Internet. Quando a Internet interligar todos os serviços de saúde, recobrindo todos os municipios do território nacional, o acesso às informaçōes epidemiológicas trará resultados positivos.

Entretanto, é preciso admitir que, mesmo num planejamento para ser desenvolvido a curto e médio prazos, não serão resolvidos os problemas de notificação e subnotificação de dados, porque se trata de uma deficiência educacional que faz parte da cultura brasileira. Mas esse quadro precisa ser mudado com investimentos no campo da educação em serviço, minimizando, assim, esse grave problema de desinformação em saúde. Não é por acaso que, nos países desenvolvidos, a exemplo dos Estados Unidos, elegeram-se como prioridade para o novo milênio projetos de melhoria da educação de sua população, maiores e continuados investimentos em ciência e tecnologia e melhor qualidade na atenção à saúde do povo.

Neste processo de integração entre a epidemiologia e a Internet, no que se refere ao aspecto inovador dessa tecnologia, os institutos de pesquisa de diversas áreas básicas de ensino e assistência em saúde desempenham um importante papel como instâncias produtoras de novos conhecimentos cientificos e informações. Ao se utilizarem da Internet, os epidemiologistas e os profissionais de saúde poderão desenvolver uma prática em serviço que servirá de referência para pesquisadores e universitários, constituindo-se numa rede de informaçōes e formações de opinião que podem gerar instrumentos de apoio adequado à fundamentação das condutas dos profissionais de saúde. Portanto, o desafio atual da epidemiologia é abrir-se para a sociedade através da democratização da informação digital, via Internet, a fim de transferir e difundir novos conhecimentos e tecnologias ao complexo atendimento à saúde.

\section{INTERNET E PESQUISA COOPERATIVA}

Quando se fala em Internet, não se pode deixar de pensar em rede de computadores interligados envolvendo universidades, institutos de pesquisas, empresas e o usuário doméstico. Na realidade, a informática já faz parte do nosso dia-a-dia, por conta da utilização generalizada de computadores em bancos, lojas de departamentos, farmácias, companhias aéreas, escolas e hospitais. Essa extraordinária expansão dos computadores ocorre em virtude do avanço tecnológico e do volumoso fluxo de informaçōes que deve ser rápido, claro, exato e confiável. Em nossos dias, segundo Fuchs (1995), não se consegue imaginar um sistema de informaçōes que dispense a informática.

Por outro lado, a informática por si só não vai resolver o problema da situação sanitária da população brasileira, objeto central da epidemiologia, ainda que apresente uma melhoria geral em alguns aspectos. Mas essa melhoria está longe de ser satisfatória. Mesmo levandose em consideração as grandes diferenças regionais, a saúde no Brasil caracteriza-se, em geral, pela presença de doenças crônico-degenerativas (neoplasias e doenças do aparelho circulatório). Esse quadro se agrava quando aliado a causas externas, como os acidentes de trânsito e homicidios, problemas maternos e perinatais e as chamadas doenças infectocontagiosas (aids, dengue, hanseniase, malária e outras). A complexidade desse perfil é propicia para o epidemiologista pesquisar e conhecer a realidade mais efetiva da saúde da população brasileira, assim como para fazer uso de tecnologias já existentes, entre elas a Internet, de modo a desenvolver novos conhecimentos e práticas que fundamentem o processo de 
transformação social mais amplo.

Para configurar a epidemiologia nessa abordagem, pode-se utilizar a Internet como ferramenta de trabalho para o desenvolvimento de pesquisa cooperativa, ou seja, uma rede cooperativa de pesquisa que se caracteriza pelo desenvolvimento de pesquisa usando a Internet, objetivando a busca de novos conhecimentos, produtos ou serviços, que serão acompanhados de forma coletiva, reunindo instituiçōes de pesquisa e serviços de saúde. A conjunção de esforços é fundamental para resgatar o conhecimento já disponível, mas que se encontra disperso em impressos mal preenchidos ou incompletos, com escrita ilegivel ou incompreensivel, pobre em termos de informação. Estes são fatores causadores de dificuldades para o epidemiologista ou o pesquisador. A rede cooperativa de pesquisa, através da Internet, visa articular competências especificas traduzidas na epidemiologia, mostrando um caminho extraordinário para a produçăo do novo conhecimento que pode representar o começo para se avançar na solução dos problemas de comunicação e informação que nos afligem hoje.

A integração entre o saber epidemiológico e a Internet, através de relações inter e transdisciplinares, pode contribuir para a melhoria dos métodos e processos de trabalho inerentes à epidemiologia, viabilizando instrumentos de pesquisa que venham beneficiar os usuários com seus resultados. Assim, em qualquer parte do Brasil, é possivel obter informaçöes sobre determinada doença, através do SINAN (Sistema de Informação Nacional em Saúde), realizar testes com as ferramentas estatisticas disponiveis, tais como o EPInfo ou SPSS, analisar as informações e apresentar conclusões capazes de contribuir para a melhoria da qualidade da saúde da população.

Dessa forma, a pesquisa em epidemiologia pode ser realizada através da cooperação entre grupos, como resultado da própria dinâmica da pesquisa. Nesse aspecto, a Internet poderá estimular a multidisciplinaridade, a aproximação entre áreas básicas e aplicadas e o enfoque de problema em saúde, envolvendo um leque muito complexo de conhecimento. A Internet irá facilitar os procedimentos da pesquisa através de links e sites, permitindo que o pesquisador vá navegando e acessando a informação que Ihe convier. Essa flexibilidade da Internet transcende as formas tradicionais de comunicação na epidemiologia.

\section{A INTERNETNO PROCESSO DE COMUNICAÇĀO EPIDEMIOLÓGICA}

A Internet virou a palavra mágica da globalização da informação. Cada vez mais, as instituições de saúde se voltam para a rede mundial de computadores, buscando causas e soluções para os problemas cotidianos, de forma compartilhada e sinergética. As facilidades que a Internet pode proporcionar ao serviço de epidemiologia são, entre outras, as seguintes: $\S$ Trabalhar através de redes cooperativas de comunicação, permitindo amplo espaço para a pesquisa nas diferentes instituições de saúde local, regional, nacional ou internacional.

- Incentivar a multidisciplinaridade, aproximando áreas e otimizando resultados.

- Contribuir para que instituiçōes públicas e privadas de saúde assumam seu papel de difusoras de conhecimentos e tecnologias avançadas na área médica.

- Adequar o perfil do profissional de saúde às mudanças necessárias para enfrentar os desafios do século XXI, através da digitalizaçăo de dados epidemiológicos, e que resultará numa grande massa de informações e conhecimentos gerada a cada dia.

Para que se possa alcançar tais objetivos, faz-se necessário equipar todos os serviços de saúde com computadores conectados à Internet, possibilitando, através de meios eletrônicos, disseminar informações e conhecimentos que contribuirão para o desenvolvimento de pesquisas, formação de profissionais e para o treinamento em serviço das equipes de saúde. Dai a importância da informatizaçăo dos serviços, pois o resultado desse trabalho gerará um impacto significativo na medida em que se efetivar a interação entre a epidemiologia e as várias redes de comunicaçăo ou entre os serviços e os pesquisadores. 
Silva (1998) aponta a necessidade de interação entre individualidade e coletividade, acrescentando que precisamos voltar a entender que só desenvolveremos um bom trabalho coletivo na medida em que formos capazes de satisfazer necessidades pessoais, individuais e coletivas. Esse é o caminho e a Internet é o canal aberto de comunicação que permite a interligação das informações epidemiológicas ao usuário do sistema de saúde em qualquer parte do planeta, permitindo dessa forma conhecer os perfis epidemiológicos de diversos grupos humanos. Pensar em Internet na epidemiologia é pensar na interação multidisciplinar.

A tentativa de associar a tecnologia da internet como instrumento de trabalho da epidemiologia visa racionalizar o tempo gasto, o registro da informação, a comunicação entre serviços e a disponibilização de relatórios para pesquisa. Pela utilização da informática, podese chegar rapidamente a uma análise da situação de uma unidade de internação ou de todo um hospital. Cabe aqui esclarecer que seu uso não é de vital importância, mas, sem dúvida, colabora para uma melhor racionalizaçăo de tempo e qualidade de análise dos dados, resultando numa maior efetividade dos serviços de atenção à saúde (Halker et al., 1992).

O sistema de comunicação por computadores não é tarefa dificil, mas em nossos serviços de saúde pública ainda precisa ser implementado colocando à disposição da rede mundial de informação dados que poderão melhorar e facilitar a comunicação. Segundo Gonçalves et al. (1995), isso significa melhor qualidade de vida, pois a comunicação é um aspecto essencial da condição humana que repercute em todas as outras áreas da vida social.

\section{CONSIDERAÇÖES FINAIS}

A Internet, como ferramenta de pesquisa, negócios, diversão e comunicação, segue mudando a ordem das coisas e revolucionando positivamente a vida de todo o mundo, inclusive na saúde pública e na epidemiologia. Mas isso não quer dizer que seja a solução para resolver todos os problemas funcionais do sistema de informação epidemiológica. Entretanto, não se pode negar que essa tecnologia, em si mesma, encerra tudo de bom e de mais moderno e maravilhoso que podemos esperar para esse novo milênio. Sem dúvida, a associação da epidemiologia com a informática e a Internet, hoje e especialmente no futuro, deve melhorar a comunicaçăo dos dados epidemiológicos e a qualidade de vida da população pelo acesso fácil, confortável e confiável que proporciona às informações em saúde.

ABSTRACT: The objective of this study is to present some theoretical reflections on Internet as an integration tool in an epidemiology service. Considering this perspective, it focuses epidemiology and Internet; Internet and cooperative research, and the importance of Internet in the epidemiological communication process. The integration between the epidemiological knowledge and the use of Internet to globalize knowledge is emphasized as one of the most important benefits this technology brings. However, it is indicated that this technology by itself is not the solution for functional problems in the health information system.

KEYWORDS: Internet, epidemiology, information in the health

RESUMEN: El presente estudio se propone presentar algunas reflexiones teóricas sobre Internet como una herramienta de integración en los servicios de epidemiologia. Dentro de esa perspectiva se focaliza la epidemiologia y la Internet; la Internet y la investigación cooperativa y, por último, la importancia de la Internet en el proceso de comunicación epidemiológica. La integración entre el saber epidemiológico y el uso de Internet para la divulgación de los conocimientos se enfatiza como uno de los mayores beneficios de esa tecnologia que globaliza la información y trascende las formas 
tradicionales de comunicación. Sin embargo, se advierte que dicha tecnologia por si sola no es la solución para resolver los problemas funcionales de los sistemas de información en la salud.

PALABRAS CLAVE: internet; epidemiologia, información en la salud

\section{REFERÊNCIAS BIBLIOGRÁFICAS:}

ARAÚJO, M. J. B. Ações de enfermagem em saúde pública e em doenças transmissiveis. Rio de Janeiro: Bezerra de Araújo, 1983.

BREILH, J.; GRANDA, E. Saúde na sociedade. São Paulo: Cortez, Rio de Janeiro: Abrasco, 1989.

FUCHS, L. O paciente quer qualidade. O mundo da saúde, São Paulo, v. 19, n. 5, p. 182 - 184, jun. 1995.

GONÇALVES, M. J.; MACEDO, E.C.; DUDUCHI, M.; CAPOVILLA, A. G.; THIERS, V. O.; CAPOVILLA, F. C. Comunicação computadorizada a serviço da saúde e da qualidade de vida. O mundo da saúde, São Paulo, v. 19, n. 4., maio, 1995. p. 145-148.

HALKER, E.; PARREIRA, F.; FERNANDES, A. L.; SAES, A. C.; CARDO, D. M.; WEY, S. B. Utilizaçăo da informática visando maior efetividade na atuação do enfermeiro epidemiologista. Acta Paul. Enf., Säo Paulo, v. 5, n. 1/4, p. 3-7, jan./dez. 1992.

SABBATINI, R. M. E. Uma nova era na medicina. Informédica. v. 1, n. 3, p. 4, jul./ago. 1993.

SILVA, M. J.P. A importância da comunicaçăo nos processos de qualidade. Nursing, v. 1, n. 1, p. 20-26, jun. 1998.

SUSSER, M.; SUSSER, E. Um futuro para epidemiologia. In.:ALMEIDA FILHO, Naomar de, (Org.) Teoria epidemiológica hoje: fundamentos, interfaces e tendências. Rio de Janeiro: FioCruz/Abrasco, 1998. p.187-211.

Recebido em outubro de 2000

Aprovado em abril de 2001 